\title{
A Neighbor-Based Approach to Identify Tuberculosis Exposure, the Kopanyo Study
}

\author{
Patrick K. Moonan, Nicola M. Zetola, James L. Tobias, Joyce Basotli, \\ Rosanna Boyd, Eleanor S. Click, Mbatshi Dima, Othusitse Fane, Alyssa M. Finlay, \\ Matsiri Ogopotse, Xiao J. Wen, Chawangwa Modongo, John E. Oeltmann
}

Contact investigation is one public health measure used to prevent tuberculosis by identifying and treating persons exposed to Mycobacterium tuberculosis. Contact investigations are a major tenet of global tuberculosis elimination efforts, but for many reasons remain ineffective. We describe a novel neighbor-based approach to reframe contact investigations.

$\Gamma$ uberculosis (TB) is a global health emergency (1). 1 The World Health Organization (WHO) End TB Strategy proposes a $90 \%$ reduction in TB incidence and $95 \%$ reduction in TB deaths by 2035 compared with 2015 (2). To reach this target, effective interventions are needed to interrupt transmission of $\mathrm{Myco}-$ bacterium tuberculosis. Contact investigations help prevent $M$. tuberculosis transmission by identifying and treating persons in close contact with persons with TB disease (3). WHO recommends tuberculosis preventive treatment (TPT) for household members of bacteriologically confirmed pulmonary TB patients to prevent progression to active TB disease (4).

Contact investigations are a major tenet of the End TB Strategy but remain ineffective for various reasons $(2,5,6)$. Many TB programs in high-burden areas limit contact investigations to household members (6). Recent studies suggest that such restrictions might miss key exposures in the community $(7,8)$. Targeted, population-based, geographic TB screening is a potential approach to augment contact investigations (9-11)

Author affiliations: Centers for Disease Control and Prevention, Atlanta, Georgia, USA (P.K. Moonan, R. Boyd, E.S. Click,

A.M. Finlay, X.J. Wen, J.E. Oeltmann); Botswana-UPenn

Partnership, Gaborone, Botswana (N.M. Zetola, M. Dima,

O. Fane, M. Ogopotse, C. Modongo); Northrop Grumman, Atlanta

(J.L. Tobias); National Tuberculosis Program, Botswana Ministry

of Health, Gaborone (J. Basotli)

DOI: https://doi.org/10.3201/eid2605.191568 but is resource and time intensive and rarely includes TPT $(11,12)$. We used population-based, molecular epidemiologic data from Botswana to investigate potential use of a neighbor-based approach for contact investigations.

\section{The Study}

During August 2012-April 2016, we enrolled participants treated for TB disease at 30 healthcare facilities in Botswana for a prospective molecular epidemiologic study, Kopanyo. In brief, Kopanyo was designed to explore potential clinical, demographic, geographic, social relationships, and $M$. tuberculosis genotypic characteristics among persons with TB $(13,14)$. We interviewed enrolled patients by using a standardized questionnaire and abstracted clinical data from medical records (13). We collected and processed sputum samples for culture and genotyped isolates with 24-locus mycobacterial interspersed repetitive unitsvariable-number tandem-repeats by using standard methods (15). We geocoded and validated the primary residence of each enrolled patient (Appendix, https:/ / wwwnc.cdc.gov/EID/article/26/5/19-1568-App1. pdf). We excluded patients without a validated primary residential geocode and those who resided in locations outside of the study area. The study area included all 11 neighborhoods in Gaborone and 3 villages in the Ghanzi District: Ghanzi, D'Kar, and Kuke.

We defined index patients as the first culture-positive pulmonary TB patient identified and started on treatment in a household. We used residence plots to identify nearest neighbors, which we defined as those who lived immediately next door, and next-nearest neighbors, which we defined as those who lived 2 doors away (Figure). We enumerated all subsequent TB cases identified by bacteriologic confirmation and clinical diagnosis within the index home, nearest-neighbor homes, and next-nearest neighbor homes. We defined 


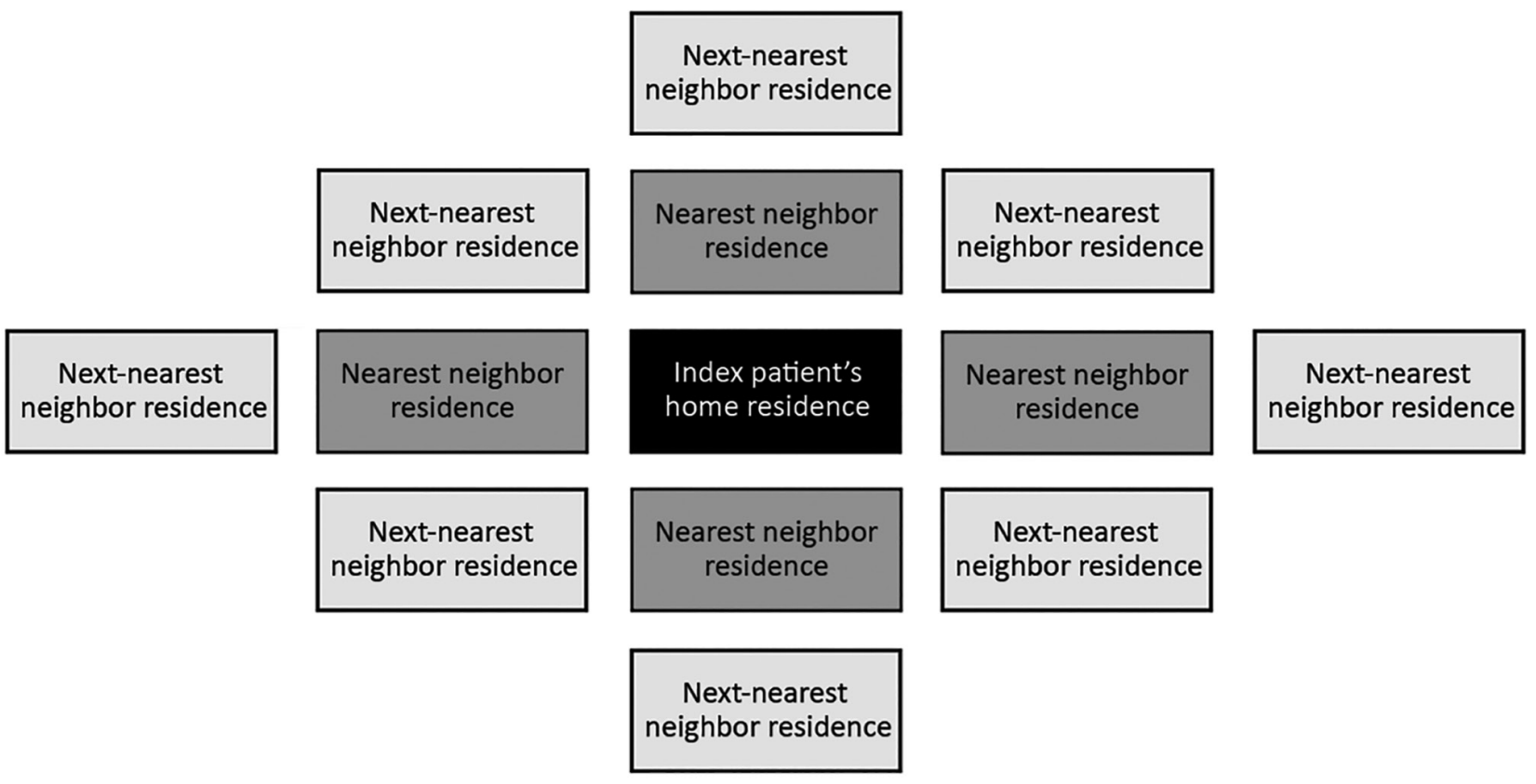

Figure. Illustration of possible nearest neighbors and next-nearest neighbors for tuberculosis (TB) screening and possible TB preventive treatment. Black box represents the home of a TB index patient; dark-gray boxes represent the nearest-neighbor homes; light-gray boxes represent the next-nearest neighbor homes. This figure does not reflect the true number of neighbor homes, and index patients might have $>4$ next-door neighbors, depending on the geographic orientation of residential plots.

future-related patients as culture-positive patients with matching genotypes diagnosed after exposure to an index patient. Concurrent disease was TB diagnosed in a contact within 90 days of the index patient.

We enrolled 4,331 patients but excluded 595 $(14 \%)$ without a residential geocode and $547(13 \%)$ who resided outside the study area. We analyzed data on the remaining 3,189 patients. Among 1,072 index patients, $143(13 \%)$ had subsequent TB patients in the home ( $\mathrm{n}=426) ; 30(7 \%)$ in-home subsequent patients had concurrent disease. Of 1,072 index patients, $73(7 \%)$ had future-related patients $(n=123)$ in their homes; 5 (3.94\%) of those had concurrent TB disease.

When we applied a neighbor-based approach, we noted that $257(24 \%)$ index patients could have subsequent TB patients living next door $(\mathrm{n}=749), 41$ of which could have concurrent disease. Among nextnearest neighbors of index patients, $390(36 \%)$ could have subsequent TB, 23 of which could have concurrent disease (Table). In addition, 29 (2.7\%) index patients could have future-related patients among their nearest neighbors $(n=42)$, and $5(0.5 \%)$ future-related patients among next-nearest neighbors $(\mathrm{n}=10), 3$ with concurrent TB disease (Table).

We found that a neighbor-based approach could identify 1,565 additional subsequent TB patients, including 175 future-related patients, and 102 patients with concurrent TB disease. The number of persons living with a bacteriologically positive patient varied by geography; however, $\approx 23,630$ contacts potentially could benefit from TPT. Of note, $9 \%(97 / 1,072)$ of index patients interviewed stated they lived alone, but $91(94 \%)$ had subsequent patients identified in the home, and $84(87 \%)$ had subsequent future-related patients living in the home.

\section{Conclusions}

We explored the use of a nearest-neighbor approach to expand TB contact investigations. This approach does not rely on name-based contact identification, which has been shown to be ineffective $(6,16-18)$. In addition, the neighbor-based approach would not require mobile screening units or mass screening campaigns in the community. By simply expanding the number of homes visited to nearest and next-nearest neighbors, the Botswana National TB Program could increase the number of TB case diagnoses by $146 \%$ and potentially interrupt 175 secondary patient transmission events.

Preventing future TB disease through TPT could also hasten TB elimination in at-risk neighborhoods and reduce deaths in the community $(11,12)$. Cegielski et al. effectively used TPT to eliminate TB from 2 atrisk neighborhoods in Texas, USA (11). The focus on nearest and next-nearest neighbors gives programs a tangible and practical approach to locating persons at risk for TB exposure and progression to TB disease. 
The neighbor-based approach differs from a neighborhood screening, which places an additional burden on TB programs by unnecessarily screening many persons at lower risk. For example, 59,100 persons reside in neighborhood C in Gaborone (data not shown). Under the neighbor-based approach, only $5,470(9 \%)$ persons, including in-home and nearest neighbor residents, would be targeted for testing.

Previous reports suggest that contact investigations fail to identify key relationships, even within households $(16,17)$. Potential stigma and lack of trust in government officials also play a role in contact investigations (16-18). In our cohort, many $(n=97)$ index patients said they lived alone, but $94 \%$ of them had subsequent cases identified in the home. In addition, $48 \%$ of future-related patients were linked to index patients who claimed no household contacts during name-based contact solicitation interviews conducted at the enrollment clinic. Household membership composition could have changed over time, and some connections might not have existed at the time of the interview. However, our study reinforces the necessity of home visits at times convenient to the index patient and when most household members are in the home, which might warrant home visits outside of business hours and flexibility in staff workplans.

Our analysis emphasizes the opportunity to prevent future TB patients and future-related TB patients by providing TPT. Household contacts, especially young children and persons living with HIV, are eligible for TPT by national policy, but TPT has not been practiced routinely in Botswana. As the Botswana Ministry of Health scales up access to TPT throughout the country, the neighbor-based approach could improve identification of most likely contacts and help target interventions where they are most needed.

Our study has limitations. Living in proximity to an index patient is not the only opportunity for transmission and might not always translate into time spent together. In addition, our analysis of futurerelated patients included only patients with culturepositive disease and genotyping results; excluding them did not affect the main analysis enumerating subsequent patients but might have underestimated the number of future-related patients. Also, our estimates for TPT represent the maximum number of persons who could benefit because we used the average number of persons per household and assumed all household members would be eligible for TPT without a reliable and available test for infection.

A neighbor-based approach should not supplant household investigations, and community-based interventions should not divert essential resources from those already devoted to finding and treating TB patients. Wide-scale implementation of this approach would require adequate resources to ensure that all patients complete the full cascade of treatment. To reach the ambitious global goal of TB elimination, we need simple, easy to implement, location-based approaches. Screening index patient households and nearest neighbors could help identify additional TB patients and persons who could benefit from TPT.

\begin{tabular}{|c|c|c|c|c|c|c|c|c|}
\hline $\begin{array}{l}\text { Geographic } \\
\text { area }\end{array}$ & $\begin{array}{l}\text { No. index } \\
\text { patients† }\end{array}$ & $\begin{array}{c}\text { No. } \\
\text { household } \\
\text { members } \\
\text { (FR) } \neq\end{array}$ & $\begin{array}{c}\text { No. nearest- } \\
\text { neighbors } \\
\text { (FR) } \ddagger\end{array}$ & $\begin{array}{l}\text { No. next- } \\
\text { nearest } \\
\text { neighbors } \\
\text { (FR)f }\end{array}$ & $\begin{array}{c}\text { Total } \\
\text { subsequent } \\
\text { patients (FR) } \ddagger\end{array}$ & $\begin{array}{c}\text { No. screened } \\
\text { to identify } 1 \\
\text { TB patient } \\
(95 \% \mathrm{Cl}) \S\end{array}$ & $\begin{array}{l}\text { Household } \\
\text { contacts that } \\
\text { could benefit } \\
\text { from TPTा }\end{array}$ & $\begin{array}{c}\text { Neighbor } \\
\text { contacts that } \\
\text { could benefit } \\
\text { from TPTा }\end{array}$ \\
\hline \multicolumn{9}{|l|}{ Gaborone } \\
\hline$A$ & 123 & 57 (16) & $93(0)$ & $47(2)$ & 197 (18) & $21(13-32)$ & 861 & 3,472 \\
\hline$B$ & 58 & $19(4)$ & $41(0)$ & $21(0)$ & $81(4)$ & $18(11-28)$ & 307 & 1,230 \\
\hline $\mathrm{C}$ & 210 & $83(22)$ & $146(8)$ & 84 (1) & $313(31)$ & $16(9-26)$ & 1,092 & 4,368 \\
\hline $\mathrm{D}$ & 195 & 58 (10) & $110(0)$ & $56(2)$ & 224 (12) & $19(11-30)$ & 878 & 3,510 \\
\hline$E$ & 79 & $28(6)$ & $46(0)$ & $30(0)$ & $104(6)$ & $11(5-20)$ & 253 & 1,011 \\
\hline $\mathrm{F}$ & 129 & $53(2)$ & $84(2)$ & $51(2)$ & $188(6)$ & $15(8-25)$ & 593 & 2,374 \\
\hline G & 51 & $14(0)$ & $29(0)$ & $18(0)$ & $61(0)$ & $9(4-17)$ & 128 & 510 \\
\hline $\mathrm{H}$ & 20 & $5(0)$ & $12(0)$ & $6(0)$ & $23(0)$ & $7(3-14)$ & 38 & 152 \\
\hline I & 6 & $2(0)$ & $9(0)^{\prime}$ & $4(0)$ & $15(0)$ & $2(0-7)$ & 10 & 41 \\
\hline J & 6 & $2(0)$ & $2(0)$ & $1(0)$ & $5(0)$ & $22(14-33)$ & 23 & 94 \\
\hline $\mathrm{K}$ & 11 & $6(0)$ & $11(0)$ & $6(0)$ & $23(0)$ & $7(3-14)$ & 35 & 141 \\
\hline \multicolumn{9}{|c|}{ Ghanzi District } \\
\hline Ghanzi & 141 & $83(57)$ & $143(24)$ & $57(3)$ & $283(84)$ & $6(2-16)$ & 398 & 1,590 \\
\hline D'kar & 35 & $9(2)$ & $14(8)$ & $7(0)$ & $30(10)^{\prime}$ & $11(5-20)$ & 86 & 280 \\
\hline Kuke & 8 & $7(4)$ & $9(0)$ & $2(0)$ & $18(4)$ & $8(3-15)$ & 28 & 128 \\
\hline Total & 1,072 & $426(123)$ & $749(42)$ & $390(10)$ & 1,565 (175) & $16(9-26)$ & 4,730 & 18,901 \\
\hline
\end{tabular}

${ }^{*} \mathrm{FR}$, future related; TB, tuberculosis; TPT, tuberculosis preventive treatment.

$\dagger$ No. index patients is equivalent to the number of standard contact investigations.

†Future related, i.e., all culture-positive patients with matching $M$. tuberculosis genotype as an index patient.

§Limits of $95 \% \mathrm{Cl}$ assume a Poisson distribution.

ๆNumber exposed to bacteriologically confirmed pulmonary TB who do not have TB disease. 
Members of the Kopanyo Study Group: Joyce Basotli, Ebi Bile, Cynthia Caiphus, Eleanor Click, Rosanna Boyd, Mbatshi Dima, Othusitse Fane, Alyssa Finlay, Sambayawo Gwebe-Nyirenda, Thandi Katlholo, Pilara Khumongwana, Chawangwa Modongo, Patrick Moonan, John Oeltmann, Matsiri Ogopotse, Kitso Ramogale, Christopher Serumola, James Shepherd, Tsaone Tamuhla, James Tobias, Goitseone Thamae, Onani Zimba, and Nicola Zetola.

\section{Acknowledgments}

We thank the tuberculosis patients and their families for participating in this study. We thank the district health team and recruitment and retention officers who helped us contact patients and coordinate clinic visits. We are indebted to the Botswana National Tuberculosis Program and Botswana Ministry of Health for their partnership in this effort, especially Botshelo Kgwaadira and Tom Lere. We also thank Tefera Agizew, Susan Cookson, Katina PappasDeLuca, Jonathan Smith, and Diya Surie for their thoughtful reviews and suggestions to improve our manuscript.

This work was funded by the National Institutes of Health (grant no. R01AI097045) and in part, by the US President's Emergency Plan for AIDS Relief through the US Centers for Disease Control and Prevention. National Institutes of Health did not participate in study design, data collection, or interpretation of results. The findings and conclusions in this report are those of the authors and do not necessarily represent the official position of the funding agencies.

\section{About the Author}

Dr. Moonan works in the Division of Global HIV and Tuberculosis, Center for Global Health, at the US Centers for Disease Control and Prevention. His research interests include tuberculosis transmission dynamics, drug-resistant tuberculosis, and applying locally relevant, operational research to influence program policy and improve program performance.

\section{References}

1. World Health Organization. Global strategy and targets for tuberculosis prevention, care and control after 2015. Geneva: The Organization; 2014.

2. Uplekar M, Weil D, Lonnroth K, Jaramillo E, Lienhardt C, Dias HM, et al.; for WHO's Global TB Programme. WHO's new end TB strategy. Lancet. 2015;385:1799-801. https://doi.org/10.1016/S0140-6736(15)60570-0

3. World Health Organization. Recommendations for investigating contacts of persons with infectious tuberculosis in low and middle-income countries. Geneva: The Organization; 2012.

4. Weis S. Contact investigations: how do they need to be designed for the 21st century? Am J Respir Crit Care Med. 2002;166:1016-7. https://doi.org/10.1164/rccm.2207007

5. Rodriguez CA, Sasse S, Yuengling KA, Azzawi S, Becerra MC, Yuen CM. A systematic review of national policies for the management of persons exposed to tuberculosis. Int J Tuberc Lung Dis. 2017;21:935-40. https:// doi.org/10.5588/ijtld.17.0061

6. Wang W, Mathema B, Hu Y, Zhao Q, Jiang W, Xu B. Role of casual contacts in the recent transmission of tuberculosis in settings with high disease burden. Clin Microbiol Infect. 2014;20:1140-5. https:// doi.org/10.1111/1469-0691.12726

7. Surie D, Fane O, Finlay A, Ogopotse M, Tobias JL, Click ES, et al.; Kopanyo Study Group. Molecular, spatial, and field epidemiology suggesting TB transmission in community, not hospital, Gaborone, Botswana. Emerg Infect Dis. 2017;23:48790. https://doi.org/10.3201/eid2303.161183

8. Moonan PK, Oppong J, Sahbazian B, Singh KP, Sandhu R, Drewyer $G$, et al. What is the outcome of targeted tuberculosis screening based on universal genotyping and location? Am J Respir Crit Care Med. 2006;174:599-604. https://doi.org/10.1164/rccm.200512-1977OC

9. Fatima R, Qadeer E, Yaqoob A, Haq MU, Majumdar SS, Shewade HD, et al. Extending 'contact tracing' into the community within a 50-metre radius of an index tuberculosis patient using Xpert MTB/RIF in Urban, Pakistan: did it increase case detection? PLoS One. 2016;11:e0165813. https://doi.org/10.1371/journal.pone.0165813

10. Cegielski JP, Griffith DE, McGaha PK, Wolfgang M, Robinson CB, Clark PA, et al. Eliminating tuberculosis one neighborhood at a time. Am J Public Health. 2013;103:1292300. https:// doi.org/10.2105/AJPH.2012.300781

11. Moonan PK, Weis SE. Assessing the impact of targeted tuberculosis interventions. Am J Respir Crit Care Med. 2008;177:557-8. https://doi.org/10.1164/ajrccm.177.5.557a

12. World Health Organization. Latent tuberculosis infection: updated and consolidated guidelines for programmatic management. Geneva: The Organization; 2018.

13. Zetola NM, Modongo C, Moonan PK, Click E, Oeltmann JE, Shepherd J, et al. Protocol for a population-based molecular epidemiology study of tuberculosis transmission in a high HIV-burden setting: the Botswana Kopanyo study. BMJ Open. 2016;6:e010046. https:/ / doi.org/10.1136/bmjopen-2015-010046

14. Click ES, Finlay A, Oeltmann JE, Basotli J, Modongo C, Boyd R, et al. Phylogenetic diversity of Mycobacterium tuberculosis in two geographically distinct locations in Botswana-the Kopanyo Study. Infect Genet Evol. 2020;81:104232 [Epub ahead of print]. https://doi.org/10.1016/j.meegid.2020.104232

15. Supply P, Allix C, Lesjean S, Cardoso-Oelemann M, Rüsch-Gerdes S, Willery E, et al. Proposal for standardization of optimized mycobacterial interspersed repetitive unitvariable-number tandem repeat typing of Mycobacterium tuberculosis. J Clin Microbiol. 2006;44:4498-510. https://doi.org/10.1128/JCM.01392-06

16. Oeltmann JE, Oren E, Haddad MB, Lake L, Harrington TA, Ijaz K, et al. Tuberculosis outbreak in marijuana users, Seattle, Washington, 2004. Emerg Infect Dis. 2006;12:1156-9. https://doi.org/10.3201/eid1207.051436

17. Asghar RJ, Patlan DE, Miner MC, Rhodes HD, Solages A, Katz DJ, et al. Limited utility of name-based tuberculosis contact investigations among persons using illicit drugs: results of an outbreak investigation. J Urban Health. 2009;86:776-80. https://doi.org/10.1007/s11524-009-9378-z

18. Nyenswah TG, Fallah M, Calvert GM, Duwor S, Hamilton ED, Mokashi V, et al. Cluster of Ebola virus disease, Bong and Montserrado Counties, Liberia. Emerg Infect Dis. 2015;21:1253-6. https://doi.org/10.3201/eid2107.150511

Address for correspondence: Patrick K. Moonan, Centers for Disease Control and Prevention, 1600 Clifton Rd NE, Mailstop US1-1, Atlanta, GA 30329-4027, USA; email: pmoonan@cdc.gov 